\title{
Professional dietary coaching within a group chat using a smartphone application for weight loss: a randomized controlled trial
}

This article was published in the following Dove Press journal: Journal of Multidisciplinary Healthcare

\author{
Kiyoji Tanaka ${ }^{1,2}$ \\ Hiroyuki Sasai ${ }^{3}$ \\ Kyohsuke Wakaba ${ }^{4}$ \\ Shin Murakami ${ }^{4,5}$ \\ Miyuki Ueda ${ }^{5}$ \\ Fumio Yamagata ${ }^{6}$ \\ Masao Sawada ${ }^{6}$ \\ Kazuhiro Takekoshi ${ }^{7}$
}

'Faculty of Health and Sport Sciences, University of Tsukuba, Tsukuba, Japan; ${ }^{2}$ THF Co., Ltd, Tsukuba, Japan; ${ }^{3}$ Department of Life Sciences, Graduate School of Arts and Sciences, The University of Tokyo, Tokyo, Japan; ${ }^{4}$ Graduate School of Comprehensive Human Sciences, University of Tsukuba, Tsukuba, Japan; ${ }^{5}$ Life Science Department, FiNC Inc., Tokyo, Japan; ${ }^{6}$ Genki Plaza Medical Center for Health Care, Tokyo, Japan; ${ }^{7}$ Faculty of Medicine, University of Tsukuba, Tsukuba, Japan
Correspondence: Kiyoji Tanaka Faculty of Health and Sport Sciences, University of Tsukuba, I-I-I Tennodai, Tsukuba, Ibaraki 305-8577, Japan Tel/fax +8I 298532655

Email tanaka.kiyoji.ft@u.tsukuba.ac.jp
Purpose: To test the effectiveness of professional dietary coaching via group chat using a smartphone application (app) for weight loss.

Methods: This study was a 12-week, assessor-blind, parallel-group, waitlist-controlled randomized trial that included a 4-week follow-up period (trial registration, UMIN000025340). Data were collected between October 2016 and May 2017 and were analyzed between July 2017 and January 2018. Participants were 112 overweight, obese, or abdominally obese Japanese adults, aged 20 to 64 years, with at least one cardiometabolic risk factor. Participants were randomized to the coaching group $(n=75)$ or control group $(n=37)$, with a ratio of $2: 1$. The coaching group received a commercial weight loss program characterizing dietary coaching by a certified nutrition professional via group chat delivered on a smartphone app. Participants posted photos of every meal into the group chat, and the certified professional gave immediate direct feedback and encouragement. The primary outcome was an 8 -week weight change. Secondary outcomes included 8-week changes in cardiometabolic risk factors. The frequency of meal photo uploads was recorded as a measure of adherence.

Results: Of the 112 randomized participants, 93 (83.0\%) and 81 (72.3\%) completed 8-week and 12 -week visits, respectively. Intention-to-treat analysis demonstrated significantly larger 8-week weight loss in the coaching group $(-1.4 \mathrm{~kg}$; 95\% confidence interval $[\mathrm{CI}]:-2.0,-0.8 \mathrm{~kg})$ than that in the control group $(-0.1 \mathrm{~kg} ; 95 \% \mathrm{CI}:-0.6,0.4 \mathrm{~kg})$. Significantly larger improvements in triglyceride and glycated hemoglobin A1c levels were also obtained in the coaching group. These benefits, except for the triglyceride level, were maintained until week 12 . The frequent upload of meal photos was associated with a larger 8-week weight loss in a dose-response fashion $(P$-value for trend $<0.001)$.

Conclusion: This smartphone-delivered commercial weight loss program characterized as dietary coaching via group chat resulted in modest but significant weight loss. Facilitating participants' active involvement in the program is necessary to achieve greater health benefits.

Keywords: specific health guidance, technology-enhanced program, meal photo upload

\section{Introduction}

Body weight loss and its maintenance remain important challenges in clinical and public health settings. ${ }^{1,2}$ Most of the clinical guidelines for obesity recommend dietary restriction as a first-line treatment option before proceeding to more intensive modalities such as pharmacotherapy and bariatric surgery. ${ }^{2-5}$ In public health practices, weight control via dietary modification is also highly recommended. In April 2008, the Japanese Ministry of Health, Labour and Welfare launched a nationwide health 
check-up and interventional program for targeting metabolic syndrome. ${ }^{1,6}$ The program is aimed at early identification of high-risk individuals through effective health check-ups, with an opportunity for lifestyle (mainly dietary) modification to prevent the development of cardiovascular disease. ${ }^{1,5}$ However, traditional weight control programs with frequent visits in face-to-face settings are often time-consuming, and labor- and cost-intensive, require large client efforts, and thus limit the widespread application to a large proportion of the target population.

With these issues in mind, technology-enhanced weight loss programs have attracted much attention in the past two decades. ${ }^{7,8}$ The technology in this context includes the use of landline phones, mobile phones, personal digital assistants, the Internet-based tools, social media, smartphone and its applications (apps), and other smart devices such as the tablet. ${ }^{8}$ Of these, the smartphone has great potential for health promotion programs because of its various functions including behavior monitoring with a built-in camera and an accelerometer as well as the convenience of the Internet connectivity, portability, and a high dissemination rate. Although numerous smartphone apps that can track food intake, physical activity, and body weight are available in the commercial app databases, such as the iOS App Store and Google Play, ${ }^{9}$ the effectiveness of the use of these smartphone apps on weight loss has rarely been tested by well-designed randomized controlled trials. ${ }^{10}$ In fact, a systematic review on smartphone-delivered weight loss interventions included a total of six trials (publications were retrieved from April 2015), but only one of them adopted an appropriately controlled trial. ${ }^{10}$ Sutton and Redman also identified remarkably few active, completed, and published trials designed to aid weight loss using a smartphone in the clinical trial registry of ClinicalTrial.gov (accessed February 5, 2016). ${ }^{11}$

There exist several other issues to be tackled in this field. Khaylis et al reported five key behavioral techniques for technology-enhanced weight loss interventions (ie, selfmonitoring, counselor feedback and communication, social support, use of a structured program, and use of an individually tailored program). ${ }^{12}$ However, no study assesses fully the advantages of the smartphone apps and how to integrate the key behavioral techniques into weight loss programs. In addition, Coughlin et al indicated the need for culturally tailored smartphone apps for weight management since most currently available apps were offered in English. ${ }^{13}$ Thus, the development and testing of non-English smartphone apps for weight loss would be valuable to widely disseminate a scientifically proven weight loss program.
One of Japan's health tech start-up companies, FiNC Inc., developed a Japanese smartphone app called "FiNC" that can upload meal photos by users who then receive immediate direct feedback by certified nutrition professionals within a group chatroom. This randomized controlled trial tested the effectiveness of a weight loss program delivered on a smartphone app among overweight or obese Japanese adults. The findings from this trial would enable us to reach a large portion of high-risk individuals at lower costs and contribute to improving population health.

\section{Methods \\ Design and setting}

This study was an assessor-blind, parallel-group, waitlistcontrolled, randomized trial comprised of an 8-week intervention and a 4-week non-intervention follow-up periods. The trial was implemented at a preventive medical care clinic in Tokyo, Japan, between October 2016 and May 2017, and data were analyzed between July 2017 and January 2018. The Ethics Review Board of the Faculty of Health and Sport Sciences at the University of Tsukuba reviewed and approved the study protocol (approval number: 28-65) on October 17, 2016. The study protocol was placed in the University Hospital Medical Information Network (UMIN) Clinical Trials Registry (UMIN000025340) on December 20, 2016. This article followed the Consolidated Standards of Reporting Trials 2010 guidelines. ${ }^{14}$ All participants gave written informed consent prior to eligibility assessment. No compensation was offered to any of the participants upon completion of this study.

Our target for enrollment was 90 participants, which allowed us to detect an effect size (Cohen's $d$ ) of 0.64 with a two-tailed alpha level of $5 \%$ and a power of $80 \% .{ }^{15}$ The effect size was determined according to our previously collected data when we provided the same weight loss program to a different group of customers. The data used for the sample size estimation are designated as confidential company information, and we are therefore unable to disclose this. Considering that some would decline to provide the informed consent or would be unable to fulfill the eligibility criteria, we attempted to invite 120 candidates for the initial eligibility assessment.

\section{Participants and randomization}

Participants were recruited from employees of several companies mainly in transportation or real estate industries in Tokyo, Japan. Through e-mail and verbal outreach, the human resources department of these companies encouraged employees who met the study eligibility listed below, 
validated by a recent health check-up result, to participate in this trial. The candidates were enrolled if they met all the following criteria: 1) Japanese adults aged 20-64 years, 2) body mass index $(\mathrm{BMI}) \geq 25 \mathrm{~kg} / \mathrm{m}^{2}$ or waist circumference $\geq 85 \mathrm{~cm}$ for male and $\geq 90 \mathrm{~cm}$ for female, 3 ) having at least one of the cardiometabolic risk factors such as elevated blood pressure (systolic blood pressure $\geq 130 \mathrm{~mm} \mathrm{Hg}$ and/or diastolic blood pressure $\geq 85 \mathrm{~mm} \mathrm{Hg}$ ), dyslipidemia (triglyceride $\geq 150 \mathrm{mg}$ / $\mathrm{dL}$ and/or high-density lipoprotein cholesterol $<40 \mathrm{mg} /$ $\mathrm{dL}$ ), and mild hyperglycemia (fasting plasma glucose $\geq 100$ $\mathrm{mg} / \mathrm{dL}$ and/or glycated hemoglobin A1c $\geq 5.2 \%$ [National Glycohemoglobin Standardization Program $]^{16}$ ) according to risk stratification criteria for the Japanese nationwide health check-ups. ${ }^{1,6}$ Since Japanese are reported to be susceptible to development of type 2 diabetes at a low level of obesity, ${ }^{17}$ the lower cutoff value of glycated hemoglobin A1c was used for early identification and intervention for high-risk individuals with type 2 diabetes. We excluded candidates if they intended to participate in or were enrolled in other clinical trials. Although those with current disease status were not excluded, we excluded candidates if they had contraindications to intentional weight loss through dietary modification judged by a physician.

After stratifying by recruitment waves, eligible participants were randomized to the coaching group or control group, with a ratio of $2: 1$. This weighted allocation ratio toward the coaching group was designed to minimize the number of control group who cannot receive dietary advices until the study end for an ethical reason. An investigator who lacked direct contact with the participants and assessors generated a random number sequence using a validated computer program.

\section{Intervention}

Participants in the coaching group received a commercial weight loss program called "Wellness Coach" developed and provided by FiNC Inc. The participants in the control group did not receive any intervention for 8 weeks or during the 4-week follow-up period. After the 12-week study period, the control participants were offered the identical weight loss program for ethical considerations.

The Wellness Coach is a weight loss program that gives users dietary support by connecting them with nutrition professionals for advice, instruction, and encouragement through the FiNC smartphone app. This weight loss program adopted four out of five established behavioral techniques for a technology-based health promotion program, ie, selfmonitoring, counselor feedback and communication, social support, and considered an individually tailored program. ${ }^{12}$ First, the participants were asked to download the app on their own smartphones and were assigned to a group with up to six members (14 groups in total) with a nutrition professional. Participants were instructed to measure their body weight twice a day in the morning and at night, according to a randomized trial of self-weighing for Japanese, ${ }^{18}$ and to take photos of their meals three times a day. Then, they uploaded the photos to a group chat system within the app which can allow users to self-monitor their weight and meals (self-monitoring). The certified nutrition professional used the group chat to give immediate and direct feedback to each participant on things such as meal choices or answers to dietary questions (counselor feedback and communication, and individually tailored program). The nutrition professionals replied to a participant's post within 3 hours during the day or by noon the next day if participants posted their photo or comment after midnight. This removed time constraints and the need for a place to receive professional dietary advice and community support. We recorded the total number of meal photo uploads as a proxy measure of study adherence and used it for sub-analyses described later. Participants were also able to communicate with other participants within their own group, but not between groups (social support). The weight monitoring records for each participant were not visible to other members due to privacy reasons. Whereas, the meal photos uploaded by the participant, the advice, comments and encouragement by the nutrition professional were disclosed to all other members for their learning purposes. The Wellness Coach program is now available primarily for corporate customers such as national, local, and corporate health insurance societies. A similar weight loss program with a one-to-one online chat system, rather than a group chatroom, is available for individual customers. This is because the effectiveness of social (peer) support on behavior change would be possibly smaller for individuals than corporate customers.

The Wellness Coach program adopted a unique diet principle called the "FiNC Method", which encouraged participants to consume carbohydrate-rich foods, protein-rich foods, and vegetables with a weight ratio of $1: 2: 3$. The portion sizes meeting this ratio every meal roughly corresponded to half a hand/palm size for carbohydrate-rich foods, a hand/ palm size for protein-rich foods, and both hands/palms size for vegetables. This simple diet principle was aimed at an easy adoption by the general population without any tools or complicated energy calculations. In practice, the nutrition professionals reviewed whether a given participant followed the diet principle. If not, the professional gave detailed advice 
on how to improve their meal choices, patterns, and other tips. The nutrition professional also evaluated the adherence for this diet principle using a five-grade scale and gave feedback to participants. No specific target for energy restriction was set for this program. Eating snacks was allowed within the nutritional balance outlined with the FiNC Method.

The nutrition professionals also applied the transtheoretical model (or stages of change) for health behavior change when providing dietary advice to participants. ${ }^{19}$ We assumed that all participants fell into preparation or action stages because they were willing to participate in our weight loss program by themselves. Participants at the preparation stage shared their reasons for participating in the Wellness Coach program and their ultimate goals with the nutrition professionals. Participants at the action stage were instructed to upload their weight and meal photos to the group chat system, and the nutrition professionals gave immediate direct feedback to them. This process targeted maintaining motivation by the immediate direct feedback and social (peer) supports or encouragement, managing reinforcement by the evaluation on the five-grade scale, and fostering a desire for mastery on dietary behavior.

Each nutrition professional was uniquely certified by FiNC Inc. Candidate professionals who possessed the right qualifications or who had a professional license, such as a dietician, nurse, pharmacist, and physician, received certification after passing the examination designated by the company. The nutrition professionals received compensation for their services according to regulations of an affiliated company.

\section{Measurements}

Demographic and lifestyle characteristics were recorded only at baseline. All study outcomes were assessed at baseline, week 8 , and week 12 by trained clinic staff who were blinded to the group allocation. The primary outcome was an 8 -week body weight change. Secondary outcomes included a 12-week weight change, 8-week changes in waist circumference, and improvement in various cardiometabolic risk factors such as blood pressure, lipid profiles, and glucose metabolism. As part of compliance outcomes, obesogenic eating behaviors were also surveyed at baseline and at week 8 .

\section{Demographic and lifestyle characteristics, and anthropometrics}

Participants reported their sex, age, sleeping status (sleep $\geq 6$ hours every day or not), and current smoking and drinking status (yes or no for both) via self-administered Web-based questionnaires. Body weight was measured to the nearest 0.1 kg using a calibrated digital scale (WB-150; Tanita, Tokyo, Japan). Height was determined to the nearest $0.1 \mathrm{~cm}$ using a portable stadiometer (AD-6227; A\&D, Tokyo, Japan) at baseline to calculate BMI. Waist circumference was measured to the nearest $0.1 \mathrm{~cm}$ at the umbilicus level using a flexible plastic tape in the standing position.

\section{Blood pressure and blood biochemistry}

Systolic and diastolic blood pressure was measured with an automated sphygmomanometer (HEM-907; Omron Healthcare, Kyoto, Japan) on the arm of seated participants who had taken sufficient rest with the arm supported at heart level. If the value for the first reading was $>130 \mathrm{~mm} \mathrm{Hg}$ for systolic or $>85 \mathrm{~mm} \mathrm{Hg}$ for diastolic, the second reading was taken after participants had had several deep breaths. When the second reading was available, the lower value of the two readings was used for analysis.

A blood sample was drawn from the antecubital vein of each participant after overnight fasting. Serum triglycerides were determined enzymatically (Determiner L TG II; Kyowa Medex, Tokyo, Japan). Serum high-density lipoprotein cholesterol and low-density lipoprotein cholesterol were measured by the direct method (MetaboLead HDL-C and LDL-C; Kyowa Medex). Glycated hemoglobin A1c was determined by high-performance liquid chromatography (TSKgelG11; Tosoh, Tokyo, Japan). The blood sample was assayed by a laboratory in the data collection site.

\section{Obesogenic eating behavior}

Participants also self-reported various obesogenic eating behaviors on the Web-based questionnaire. They reported whether they had a history of eating snacks, eating out, eating fast foods (such as "hamburgers, french-fried potatoes, or fried chicken"), eating confectioneries, drinking sugarsweetened beverages ( $\geq$ once a week or less, for these five items), eating until full, and binge eating (yes or no, for both). The cutoff point of these question items was selected by referring to a previous study of eating behavior. ${ }^{20}$

\section{Statistical analysis}

All statistical analyses were complied with the analysis plan in the study protocol. The data were analyzed by IBM SPSS Statistics for Windows, version 22.0 (IBM, Armonk, NY, USA), with the two-tailed significance level set at 5\%. Participant baseline characteristics were summarized as mean and standard deviation for continuous variables or percentage for categorical variables. The triglyceride level was presented as median and interquartile range and was log-transformed 
for analyses hereafter. The primary analysis followed an intention-to-treat (ITT) principle, with missing data imputed by the baseline observations carried forward rule. Primary, secondary, and compliance outcomes (ie, changes from baseline) were expressed with mean and $95 \%$ confidence interval (CI). To test the superiority of the coaching group over the control group, unpaired $t$-tests were employed to compare primary and secondary outcomes between the two groups. To compare improvement rates in eating behaviors between the two group, a $\chi^{2}$-test was also applied.

To explore the moderating effect of participants' age and baseline BMI on weight loss, we tested group-by-age ( $<$ or $\geq$ the median age of 46 years) and group-by-BMI ( $<$ or $\geq$ the median BMI of $27.0 \mathrm{~kg} / \mathrm{m}^{2}$ ) interactions using a two-way analysis of variance in separate models. Next, to explore dose-response associations of adherence to the weight loss program with study outcomes in the coaching group, we performed linear trend tests within linear regression models. In these models, we treated study outcomes as dependent variables; tertiles for the frequency of meal photo uploads to the group chat system (coded as 1, 2, and 3 ) as the primary exposure variable; and age, baseline
BMI, and the baseline value for each dependent variable as covariates.

\section{Results}

The participant flow is illustrated in Figure 1. Following the recruitment efforts, 116 candidates provided written informed consent and were invited to a baseline examination. Contrary to our expectation, a larger proportion of candidates $(n=112)$ fulfilled the eligibility criteria. The 112 eligible adults were randomly allocated to the coaching $(n=75)$ or control groups $(\mathrm{n}=37)$. The 112 randomized participants were included for the ITT analysis. Of the 112 randomized participants, 93 $(83.0 \%)$ and $81(72.3 \%)$ completed 8 -week and 12-week visits, respectively. The retention rates in the coaching group were slightly lower than those in the control group $(80.0 \%$ vs $89.2 \%$ at week $8 ; 68.0 \%$ vs $81.1 \%$ at week 12 ), but they did not statistically differ between the two groups at both week $8(P=0.22)$ and week $12(P=0.14)$. The detailed reasons for missed assessment were mostly scheduling conflicts and lost to follow-up including retirement from the companies.

Baseline participant characteristics are summarized in Table 1. No pronounced differences existed between the two

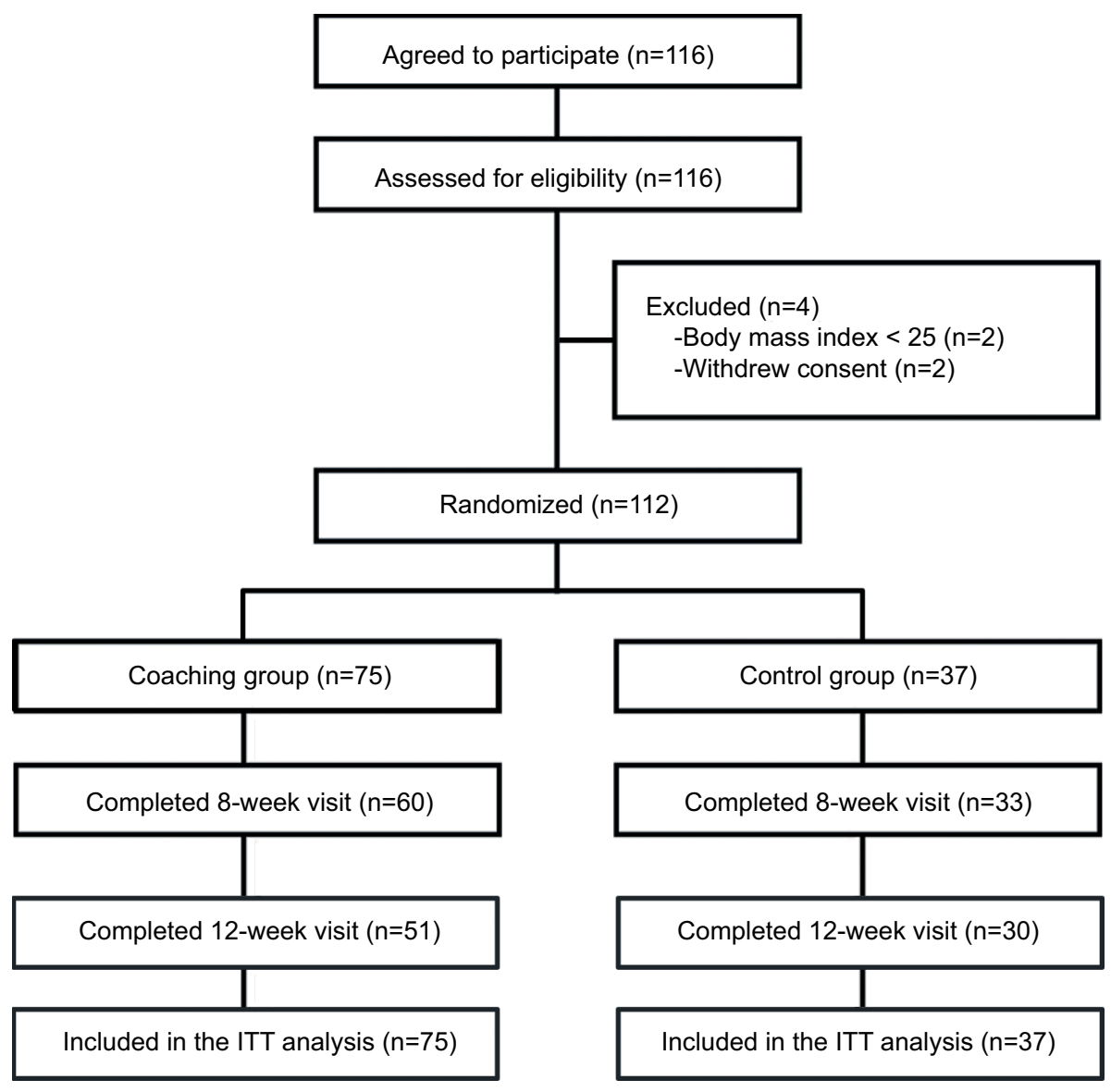

Figure I Participant flowchart.

Abbreviation: ITT, intention-to-treat. 
Table I Participant characteristics at baseline

\begin{tabular}{|c|c|c|}
\hline Characteristics & $\begin{array}{l}\text { Coaching } \\
\text { group } \\
(n=75)\end{array}$ & $\begin{array}{l}\text { Control } \\
\text { group } \\
(n=37)\end{array}$ \\
\hline \multicolumn{3}{|l|}{ Demographics and lifestyle } \\
\hline Female & $1.3 \%$ & $0.0 \%$ \\
\hline Age, years & $45.6(10.2)$ & $47.8(9.3)$ \\
\hline Sleeping $\geq 6$ hours every day* & $14.1 \%$ & $15.2 \%$ \\
\hline Current smoking* & $46.5 \%$ & $30.3 \%$ \\
\hline Current alcohol drinking* & $71.8 \%$ & $72.7 \%$ \\
\hline \multicolumn{3}{|l|}{ Anthropometrics } \\
\hline Height, cm & I $72.3(5.9)$ & I $72.0(5.5)$ \\
\hline Weight, kg & $83.1(11.1)$ & $83.5(9.8)$ \\
\hline Body mass index, $\mathrm{kg} / \mathrm{m}^{2}$ & $28.0(3.3)$ & $28.2(3.0)$ \\
\hline Waist circumference, $\mathrm{cm}$ & $97.7(9.1)$ & $98.3(7.5)$ \\
\hline \multicolumn{3}{|l|}{ Blood pressure, mm Hg } \\
\hline Systolic & I30.9 (|3.2) & | $30.8(\mid 4.9)$ \\
\hline Diastolic & $82.7(12.1)$ & $82.9(11.3)$ \\
\hline \multicolumn{3}{|l|}{ Blood biochemistry } \\
\hline Triglycerides, mg/dL & $146.0(126.5)$ & $146.0(94.0)$ \\
\hline HDL cholesterol, mg/dL & $52.1(11.5)$ & $52.4(10.8)$ \\
\hline LDL cholesterol, mg/dL & I35.4 (31.2) & I35.6 (34.3) \\
\hline Glycated hemoglobin Alc, \% & $5.97(0.8 \mathrm{I})$ & $5.87(0.65)$ \\
\hline \multicolumn{3}{|l|}{ Obesogenic eating behavior* } \\
\hline Eating snacks $\geq$ once a week & $71.8 \%$ & $72.7 \%$ \\
\hline Eating out $\geq$ once a week & $94.4 \%$ & $90.9 \%$ \\
\hline Eating fast foods $\geq$ once a week & $38.0 \%$ & $33.3 \%$ \\
\hline Eating confectioneries $\geq$ once a week & $77.5 \%$ & $87.9 \%$ \\
\hline Drinking SSBs $\geq$ once a week & $63.4 \%$ & $63.6 \%$ \\
\hline Eating until full & $84.5 \%$ & $93.9 \%$ \\
\hline Binge eating & $56.3 \%$ & $54.5 \%$ \\
\hline
\end{tabular}

Notes: Data expressed as mean (standard deviation) unless specified. The triglyceride presented as median (interquartile range). *Data available for 71 (94.7\%) participants in the coaching group and $33(89.1 \%)$ in the control group.

Abbreviations: HDL, high-density lipoprotein; LDL, low-density lipoprotein; SSBs, sugar-sweetened beverages.

groups. A female participant was included in the coaching group. The median (first-third quartiles) frequency of meal photo uploads per participant was 82 times (23-139.5 times) during the 8-week period. The frequencies of photo uploads every 10 days per participant were 17 times (5.5-25.5 times) for the initial 10 days, 16 times (4-28 times) for days 11 to 20,17 times (1-25 times) for days 21 to 30,17 times ( $0-25$ times) for days 31 to 40,15 times ( $0-25.5$ times) for days 41 to 50 , and 3 times ( $0-18.5$ times) for day 51 to the end of the program.

Our primary ITT analyses in Table 2 demonstrated significantly larger weight loss over 8 weeks in the coaching group than that in the control group. Significant between-group differences were also obtained for triglyceride levels and glycated hemoglobin A1c levels. These beneficial effects, except for the triglyceride level, essentially remained stable after the 4-week non-intervention period. The 12-week reduction in waist circumference was also larger in the coaching group than in the control group. The moderation analyses revealed that neither participants' age nor baseline BMI altered the weight changes in response to interventions (group-by-age interaction, $P=0.32$; group-by-BMI interaction, $P=0.90$ ). We found beneficial associations between the frequent upload of meal photos and changes in body weight, waist circumference, and glycated hemoglobin A1c level in a dose-response manner ( $P$-value for trend $<0.05$ for all). As compared to the first tertile ( $<29$ times) of the photo upload frequency, weight loss for the second (29-127 times) and the third ( $\geq 128$ times) tertiles exhibited $1.9 \mathrm{~kg}$ (95\% CI: 0.6, $3.2 \mathrm{~kg})$ and $3.5 \mathrm{~kg}(95 \%$ CI: $2.2,4.8 \mathrm{~kg}$ ), respectively. Waist circumference reduced by $3.2 \mathrm{~cm}(95 \%$ CI: $1.4,4.9 \mathrm{~cm})$ for the second tertile and $3.9 \mathrm{~cm}(95 \% \mathrm{CI}: 2.1,5.7 \mathrm{~cm})$ for the third tertile. Similarly, changes in glycated hemoglobin A1c were - $0.09 \%$ (95\% CI: $-0.28,0.11 \%)$ for the second tertile and $-0.26 \%(95 \% \mathrm{CI}$ : $-0.46,-0.07 \%$ ) for the third tertile.

\section{Discussion}

This randomized trial of 112 overweight or obese adults tested the effectiveness of a commercial weight loss program that featured professional coaching within the group chat function of the smartphone app. We identified the following key findings: 1) weight loss over 8 weeks in the coaching group was $1.3 \mathrm{~kg}$ larger than that in the waitlist control group, 2) triglyceride and glycated hemoglobin A1c levels improved to a greater extent in the coaching group, 3) these modest but significant benefits, except for the triglyceride level, were maintained after the 4-week non-intervention period, 4) the 12-week reduction in waist circumference was significantly larger in the coaching group, 5) participants who uploaded their meal photos more often gained greater benefits than those who did it less often.

The weight loss amount of $1.4 \mathrm{~kg}$ seemed somewhat lower than those of previous technology-enhanced weight loss programs. A systematic review summarizing the findings from 27 technology-assisted weight loss programs (ie, any types of technology included) reported a weight loss of 0.6 to $11.3 \mathrm{~kg}$ in durations ranging from 8 weeks to 24 months. ${ }^{8}$ Another systematic review assessing mobile-technology (ie, mobile phone and portable digital assistant) interventions for weight loss included seven randomized controlled trials, which demonstrated weight loss amounts that ranged from 3.9 to $11.8 \mathrm{~kg}$ during 8 to 52 weeks. ${ }^{21}$ A systematic review exclusively on smartphone-app-based interventions identified six studies including single-arm, non-randomized 
Table 2 Intention-to-treat analyses for changes in the primary and secondary outcomes, and percent improvement for eating behaviors

\begin{tabular}{|c|c|c|c|c|c|c|}
\hline \multirow[t]{2}{*}{ Dependent variables } & \multicolumn{3}{|c|}{ 8-week changes or $\%$ of those improved } & \multicolumn{3}{|l|}{ I 2-week changes } \\
\hline & $\begin{array}{l}\text { Coaching group } \\
(n=75)\end{array}$ & $\begin{array}{l}\text { Control group } \\
(n=37)\end{array}$ & $P$-values & $\begin{array}{l}\text { Coaching group } \\
(n=75)\end{array}$ & $\begin{array}{l}\text { Control group } \\
(n=37)\end{array}$ & $P$-values \\
\hline \multicolumn{7}{|l|}{ Anthropometrics } \\
\hline Weight, kg & $-1.4(-2.0,-0.8)$ & $-0.1(-0.6,0.4)$ & 0.001 & $-1.4(-2.1,-0.8)$ & $-0.1(-0.7,0.6)$ & 0.004 \\
\hline Waist circumference, $\mathrm{cm}$ & $-1.9(-2.8,-1.0)$ & $-0.9(-2.0,0.3)$ & 0.15 & $-2.8(-3.6,-1.9)$ & $-1.1(-2.3,0.2)$ & 0.02 \\
\hline \multicolumn{7}{|l|}{ Blood pressure, mm Hg } \\
\hline Systolic & $-4.0(-6.6,-1.4)$ & $-1.6(-5.4,2.2)$ & 0.30 & $-1.9(-4.4,0.5)$ & I.2 $(-2.6,4.9)$ & 0.17 \\
\hline Diastolic & $-3.7(-5.5,-1.9)$ & $-1.7(-4.7,1.2)$ & 0.25 & $-2.2(-4.1,-0.4)$ & $0.1(-2.9,3.1)$ & 0.19 \\
\hline \multicolumn{7}{|l|}{ Blood biochemistry } \\
\hline Log triglycerides, mg/dL & $-0.11(-0.20,-0.02)$ & $0.11(-0.03,0.26)$ & 0.01 & $-0.05(-0.15,0.06)$ & $0.15(-0.03,0.33)$ & 0.06 \\
\hline HDL cholesterol, mg/dL & $-I . I(-2.6,0.4)$ & $-1.7(-3.3,-0.1)$ & 0.57 & $0.1(-1.6,1.7)$ & $-0.1(-2.7,2.6)$ & 0.94 \\
\hline LDL cholesterol, mg/dL & $-4.0(-8.4,0.5)$ & $0.2(-6.7,7.0)$ & 0.31 & $-4.8(-8.4,-1.2)$ & $-3.5(-10.8,3.7)$ & 0.75 \\
\hline Glycated hemoglobin Alc, \% & $-0.15(-0.24,-0.06)$ & $0.02(-0.06,0.10)$ & 0.01 & $-0.11(-0.21,-0.02)$ & $0.01(-0.06,0.07)$ & 0.04 \\
\hline \multicolumn{7}{|l|}{ Obesogenic eating behavior* } \\
\hline Eating snacks $\geq$ once a week & $16.9 \%$ & $6.1 \%$ & 0.23 & - & - & \\
\hline Eating out $\geq$ once a week & $4.2 \%$ & $3.0 \%$ & 1.00 & - & - & \\
\hline Eating fast foods $\geq$ once a week & $19.7 \%$ & $6.1 \%$ & 0.13 & - & - & \\
\hline $\begin{array}{l}\text { Eating confectioneries } \geq \text { once } \\
\text { a week }\end{array}$ & $8.5 \%$ & $3.0 \%$ & 0.54 & - & - & \\
\hline Drinking SSBs $\geq$ once a week & $14.1 \%$ & $3.0 \%$ & 0.17 & - & - & \\
\hline Eating until full & $14.1 \%$ & $6.1 \%$ & 0.39 & - & - & \\
\hline Binge eating & $23.9 \%$ & $12.1 \%$ & 0.26 & - & - & \\
\hline
\end{tabular}

Notes: Data expressed as mean ( $95 \%$ confidence interval) unless specified. ${ }^{*}$ The denominators were 71 in the coaching group and 33 in the control group. Abbreviations: HDL, high-density lipoprotein; LDL, low-density lipoprotein; SSBs, sugar-sweetened beverages; -, not assessed.

and randomized controlled trials and demonstrated a 0.03 to $10.9 \mathrm{~kg}$ of weight loss during periods ranging from 8 to 24 weeks. ${ }^{10}$ Moreover, two recent randomized trials, which were not included in the previously-mentioned systematic reviews, ${ }^{8,10,17}$ showed 4.0 and $6.4 \mathrm{~kg}$ of weight loss for 6 months. ${ }^{22,23}$ Collectively, the weight loss achieved with our program was relatively lower than those from other studies. This indicates that there is ample room for further improvement and refinement in our program.

The modest weight loss observed in this trial may be accounted for in part by fewer participants that were involved in the program (ie, constant login and meal photo uploads) than our expectation. Without constant logins and meal photo uploads, the nutrition professionals had no opportunity to provide participants with effective dietary advice. The median photo upload was 82 times during the 8 -week period, which corresponded to less than half of the full opportunities (3 meals per day $\times 8$ weeks). In the latter half of the program (ie, weeks 5 to 8 ), one-fourth or more of the participants did not upload any meal photos in each 10-day interval. The frequency of meal photo uploads was beneficially associated with weight loss. Furthermore, a retrospective study for users of a smartphone-based commercial weight loss app named
"Noom Coach" identified meal input frequency into the app as a strong predictor for weight loss. ${ }^{24}$ Thus, facilitating an active involvement to the app, ie, friendly reminder for those with no recent photo uploads, can be a key measure to improve the health benefits from our program.

Additionally, several other challenges inherent in our program may explain the modest weight loss. First, meal photo-estimated portion sizes and the number of dishes might not be sufficiently accurate due to technological limitations and limitation of the number of photos as well as users' intention not to record all what they ate to imitate better eating habit. Inaccurate input, primarily due to underreporting of dietary intake, to the certified professionals could result in less effective dietary advice which may have influenced behavior change. Second, personalized advice mainly on meal choices and dietary patterns or preferences might not contribute to consistent and substantial negative energy balance. As suggested by several clinical guidelines for obesity, ${ }^{2-5}$ more strict goal setting for energy restriction might be needed to achieve clinically meaningful weight loss. Third, self-monitoring daily food intake alone might be insufficient for weight loss. Other behavioral factors such as physical activity and sleeping should have been continuously 
monitored, and feedback for participants may have been effective. The above-mentioned reasons may have collectively influenced the modest weight loss in this trial.

Nonetheless, our weight loss program elicited a significantly larger reduction in waist circumference than the control group at week 12. Increased waist circumference represents central obesity and is well known to be associated with incident stroke and mortality from all causes and cardiovascular diseases in Japanese populations. ${ }^{25,26}$ A previous study also demonstrated that gain in waist circumference was a significant predictor for the development of type 2 diabetes among Japanese adults living in urban areas, ${ }^{27}$ which resembles the target population of our trial. A clinical guideline for obesity in Japan recommends a reduction in waist circumference by $\geq 3.0 \mathrm{~cm}$ for improving cardiometabolic risk factors. ${ }^{5}$ Collectively, the modest but significant reduction in waist circumference $(2.8 \mathrm{~cm})$ through our weight loss program might lead to decreased risks for developing type 2 diabetes and prevention of cardiovascular diseases.

There are several noteworthy strengths of this study. First, this trial utilized the research design of an assessorblind, parallel-group, waitlist-controlled, randomized trial. A systematic review on smartphone-based interventions and a commentary by Sutton and Redman indicated that few randomized controlled trials currently exist. ${ }^{10,11}$ Therefore, the findings are highly reliable and will extend the current body of knowledge in this field. Second, our weight loss program gives a platform for participants to upload their meal photos and to have an easy access to their coach essentially anytime of the day. This type of approach, rather than faceto-face interventions with frequent visits, may remove time constraints and decrease costs, and can be easily adopted by wider populations such as full-time workers and students. Third, the inclusion criteria in this trial followed those for the target populations of a nationwide interventional program named "specific health guidance" in Japan. ${ }^{1,6}$ Therefore, our weight loss program can be one of the choices for nationwide health promotion practices.

In contrast, we must also mention some limitations. First, almost all participants were male contrary to the target population of both men and women. This is because the personnel of the human resources departments hesitated to actively encourage female employees to participate in our trial due to harassment concerns. Thus, the findings from this study cannot be extrapolated to female adults. In contrast, the data primarily on males remain scarce in this research field ${ }^{9}$ and thus still worth reporting. Second, the follow-up period of our weight loss program was relatively shorter (4 weeks) than the recommended duration ( $\geq 12$ months) by the clinical obesity guidelines..$^{2-4}$ Long-term effectiveness or sustainability of the obtained benefits is therefore largely unknown. After resolving several challenges identified through this trial, we would like to design another weight-loss randomized trial with a longer follow-up period ( $\geq 12$ months). Third, contrary to the recommendations by several clinical guidelines for obesity, ${ }^{2-4}$ the Wellness Coach program addressed mainly dietary behavior to achieve weight loss benefits. Incorporating other behavioral components (ie, physical activity and sleep) might lead to further refinement of this weight loss program to gain more health benefits. Fourth, we did not take any measures to avoid contamination of the intervention effects within the same company. This might contribute to the modest difference in outcome measures between the two groups. Fifth, this study did not assess detailed dietary intake such as energy and macro- and micro-nutrient intakes. Detailed assessment of dietary intake may not substantially affect study conclusions but will help in further evaluation and refinement of the weight loss program. Sixth, we had no information on prescribed medications and socioeconomic status (ie, type of employment, educational attainment, and income level), and therefore we do not know how these factors affected our study outcomes. However, since the randomization procedure theoretically balances out these characteristics between the two groups, their influences would be minimal.

\section{Conclusion}

This 8-week commercial weight loss program characterized as dietary coaching via group chat using a smartphone app resulted in modest but significant weight loss. The amount of weight loss achieved in this study was relatively lower than that reported by previous studies; however, the more frequent the meal records, the better was the achievement shown. Therefore, further improvement and refinement of our program has the potential to produce greater health benefits in Web-based settings. Further studies are needed for program improvement and refinement. Facilitating participants' active involvement in the program, including meal photo uploads (eg, sending reminders to those without recent photo uploads), may become a key process to address these challenges.

\section{Acknowledgments}

This trial was financially supported by a collaborative research agreement between Faculty of Health and Sport Sciences at the University of Tsukuba and FiNC Inc, and a research contract between FiNC Inc and THF Co., Ltd. Genki Plaza 
Medical Center for Health Care also financially supported part of the costs related to baseline and follow-up assessments. We gratefully acknowledge the contribution of all the trial staff.

\section{Disclosure}

$\mathrm{K}$ Tanaka is the president of THF Co., Ltd. S Murakami and M Ueda are employees of FiNC Inc. F Yamagata and M Sawada are staff members at the Genki Plaza Medical Center for Health Care. The authors report no other conflicts of interest in this work.

\section{References}

1. Tsushita K, Hosler AS, Miura K, et al. Rationale and descriptive analysis of specific health guidance: the nationwide lifestyle intervention program targeting metabolic syndrome in Japan. J Atheroscler Thromb. 2018;25(4):308-322.

2. Jensen MD, Ryan DH, Apovian CM, et al.; American College of Cardiology/American Heart Association Task Force on Practice Guidelines.; Obesity Society. 2013 AHA/ACC/TOS guideline for the management of overweight and obesity in adults: a report of the American College of Cardiology/American Heart Association Task Force on Practice Guidelines and The Obesity Society. Circulation. 2014;129(25 Suppl 2):S102-S138.

3. Kushner RF, Ryan DH. Assessment and lifestyle management of patients with obesity: clinical recommendations from systematic reviews. JAMA. 2014;312(9):943-952.

4. National Health and Medical Research Council. Clinical practice guidelines for the management of overweight and obesity in adults, adolescents and children in Australia. Melbourne: NHMRC; 2013 Available from: https://www.nhmrc.gov.au/files_nhmrc/publications/attachments/ n57_obesity_guidelines_140630.pdf. Accessed March 5, 2018.

5. Japan Society for the Study of Obesity, editor. Guidelines for the Management of Obesity Disease 2016. Tokyo: Life Science Publishing; 2016. Japanese.

6. Ministry of Health, Labour and Welfare. Specific health checkups and specific health guidance. Available from: http://www.mhlw.go.jp/ english/wp/wp-hw3/dl/2-007.pdf. Accessed January 31, 2018.

7. Kozak AT, Buscemi J, Hawkins MA, et al. Technology-based interventions for weight management: current randomized controlled trial evidence and future directions. J Behav Med. 2017;40(1):99-111.

8. Raaijmakers LC, Pouwels S, Berghuis KA, Nienhuijs SW. Technologybased interventions in the treatment of overweight and obesity: a systematic review. Appetite. 2015;95:138-151.

9. Chen J, Cade JE, Allman-Farinelli M. The most popular smartphone apps for weight loss: a quality assessment. JMIR mHealth uHealth. 2015;3(4):e104.

10. Semper HM, Povey R, Clark-Carter D. A systematic review of the effectiveness of smartphone applications that encourage dietary selfregulatory strategies for weight loss in overweight and obese adults. Obes Rev. 2016;17(9):895-906.
11. Sutton EF, Redman LM. Smartphone applications to aid weight loss and management: current perspectives. Diabetes Metab Syndr Obes. 2016;9:213-216.

12. Khaylis A, Yiaslas T, Bergstrom J, Gore-Felton C. A review of efficacious technology-based weight-loss interventions: five key components. Telemed J E Health. 2010;16(9):931-938.

13. Coughlin SS, Hardy D, Caplan LS. The need for culturally-tailored smartphone applications for weight control. J Ga Public Health Assoc. 2016;5(3):228-232.

14. Schulz KF, Altman DG, Moher D; CONSORT Group. CONSORT 2010 statement: updated guidelines for reporting parallel group randomized trials. Ann Intern Med. 2010;152(11):726-732.

15. Faul F, Erdfelder E, Lang AG, Buchner A. G*Power 3: a flexible statistical power analysis program for the social, behavioral, and biomedical sciences. Behav Res Methods. 2007;39(2):175-191.

16. Little RR, Rohlfing CL, Sacks DB; National Glycohemoglobin Standardization Program (NGSP) Steering Committee. Status of hemoglobin A1c measurement and goals for improvement: from chaos to order for improving diabetes care. Clin Chem. 2011;57(2):205-214.

17. Takeno K, Tamura Y, Kawaguchi M, et al. Relation between insulin sensitivity and metabolic abnormalities in Japanese men with BMI of 23-25 kg/m². J Clin Endocrinol Metab. 2016;101(10):3676-3684.

18. Oshima Y, Matsuoka Y, Sakane N. Effect of weight-loss program using self-weighing twice a day and feedback in overweight and obese subject: a randomized controlled trial. Obes Res Clin Pract. 2013;7(5):e361-366.

19. Prochaska JO, Velicer WF. The transtheoretical model of health behavior change. Am J Health Promot. 1997;12(1):38-48.

20. Mitchell JE, King WC, Courcoulas A, et al. Eating behavior and eating disorders in adults before bariatric surgery. Int J Eat Disord. 2015;48(2):215-222.

21. Bacigalupo R, Cudd P, Littlewood C, Bissell P, Hawley MS, Buckley Woods H. Interventions employing mobile technology for overweight and obesity: an early systematic review of randomized controlled trials. Obes Rev. 2013;14(4):279-291.

22. Ross KM, Wing RR. Impact of newer self-monitoring technology and brief phone-based intervention on weight loss: a randomized pilot study. Obesity (Silver Spring). 2016;24(8):1653-1659.

23. Spring B, Pellegrini CA, Pfammatter A, et al. Effects of an abbreviated obesity intervention supported by mobile technology: The ENGAGED randomized clinical trial. Obesity (Silver Spring). 2017;25(7): 1191-1198.

24. Chin SO, Keum C, Woo J, et al. Successful weight reduction and maintenance by using a smartphone application in those with overweight and obesity. Sci Rep. 2016;6:34563.

25. Furukawa Y, Kokubo Y, Okamura T, et al. The relationship between waist circumference and the risk of stroke and myocardial infarction in a Japanese urban cohort: the Suita Study. Stroke. 2010;41(3):550-553.

26. Saito I, Kokubo Y, Kiyohara Y, et al. Prospective study on waist circumference and risk of all-cause and cardiovascular mortality: pooled analysis of Japanese community-based studies. Circ J. 2012;76(12):2867-2874.

27. Tatsumi Y, Watanabe M, Nakai M, et al. Changes in waist circumference and the incidence of type 2 diabetes in community-dwelling men and women: The Suita Study. J Epidemiol. 2015;25(7):489-495.
Journal of Multidisciplinary Healthcare

\section{Publish your work in this journal}

The Journal of Multidisciplinary Healthcare is an international, peerreviewed open-access journal that aims to represent and publish research in healthcare areas delivered by practitioners of different disciplines. This includes studies and reviews conducted by multidisciplinary teams as well as research which evaluates the results or conduct of such teams or health care processes in general. The journal covers a very wide range of areas and welcomes submissions from practitioners at all levels, from all over the world. The manuscript management system is completely online and includes a very quick and fair peer-review system. Visit http://www.dovepress.com/ testimonials.php to read real quotes from published authors. 